Ben-Esmael, M, Hart, B and Hayatleh, K

CMRR-Bandwidth Extension Technique for CMOS Differential Amplifiers

Ben-Esmael, M, Hart, B and Hayatleh, K (2014) CMRR-Bandwidth Extension Technique for CMOS Differential Amplifiers. AEÜ International Journal of Electronics and Communications / Archiv für Elektronik und Übertragungstechnik, 68 (10). pp. $990-993$.

doi: 10.1016/j.aeue.2014.04.022

This version is available: https://radar.brookes.ac.uk/radar/items/5ad32a31-939d-4983-98df-66bbd86ec9ec/1/

Available on RADAR: July 2016

Copyright $(C$ and Moral Rights are retained by the author(s) and/ or other copyright owners. A copy can be downloaded for personal non-commercial research or study, without prior permission or charge. This item cannot be reproduced or quoted extensively from without first obtaining permission in writing from the copyright holder(s). The content must not be changed in any way or sold commercially in any format or medium without the formal permission of the copyright holders.

This document is the post print version of the journal article. Some differences between the published version and this version may remain and you are advised to consult the published version if you wish to cite from it. 


\title{
CMRR-Bandwidth Extension Technique for CMOS Differential Amplifiers
}

\author{
M. Ben-Esmael, B. L. Hart, K. Hayatleh and F. J. Lidgey \\ Oxford Brookes University, Wheatley Campus, Oxford, OX33 1HX, UK \\ khayatleh@,brookes.ac.uk
}

\begin{abstract}
An exemplary design demonstrates how to extend the common-mode rejection ratio (CMRR) bandwidth of a CMOS differential amplifier. The design presented uses MOSFETs with a channel length of $180 \mathrm{~nm}$. A novel circuit technique is employed that partially compensates for the output capacitance of the tail current sink, thereby more than quadrupling the CMRR bandwidth in the example considered.
\end{abstract}

Keywords: (MOSFET differential amplifier, Common-mode-rejection-ratio, Bandwidth)

\section{Introduction}

The primary function of a differential amplifier is to produce an output signal that is a linearly amplified version of the normally small difference between two input signals, while rejecting the larger part of the two input signals that are common to both of them. The extent to which it is able to do this successfully is quantified by the 'Common-ModeRejection-Ratio' (CMRR), an important parameter in differential amplifiers for many applications, particularly in medical instrumentation [1]. The most commonly encountered common-mode voltage is line or mains interference, at $50 \mathrm{~Hz}$ or $60 \mathrm{~Hz}$. However, with increasing use of switched-mode power supplies and other higher frequency generators, good CMRR at higher frequencies is becoming more important to reduce the amplitude of high frequency common-mode signals in precision instrumentation applications. There have been many improvements to the classical differential pair amplifier [2-4] that improve the CMRR. However, almost all increasing the low frequency CMRR by reducing the common-mode gain, but little has been published to date to address the need for higher CMRR bandwidth performance. This paper outlines a circuit technique that specifically addresses this issue by reducing the tail sink-current capacitance significantly, resulting in a substantial increase in the CMRR bandwidth. Simulation results for an exemplary MOSFET source- coupled differential design, illustrate the advantage of the technique. It produces a four-fold CMRR bandwidth improvement.

\section{Normal Circuit Operation}

The amplifier shown in Fig.1 is a standard type of MOSFET directly-coupled differential amplifier, appropriately labelled for the discussion that follows. It is well-known that such a source-coupled differential pair amplifier provides good performance provided $M_{1}$ and $M_{2}$ are well-matched. That is achievable with good IC design techniques, such as the use of common-centroid layout methodology.

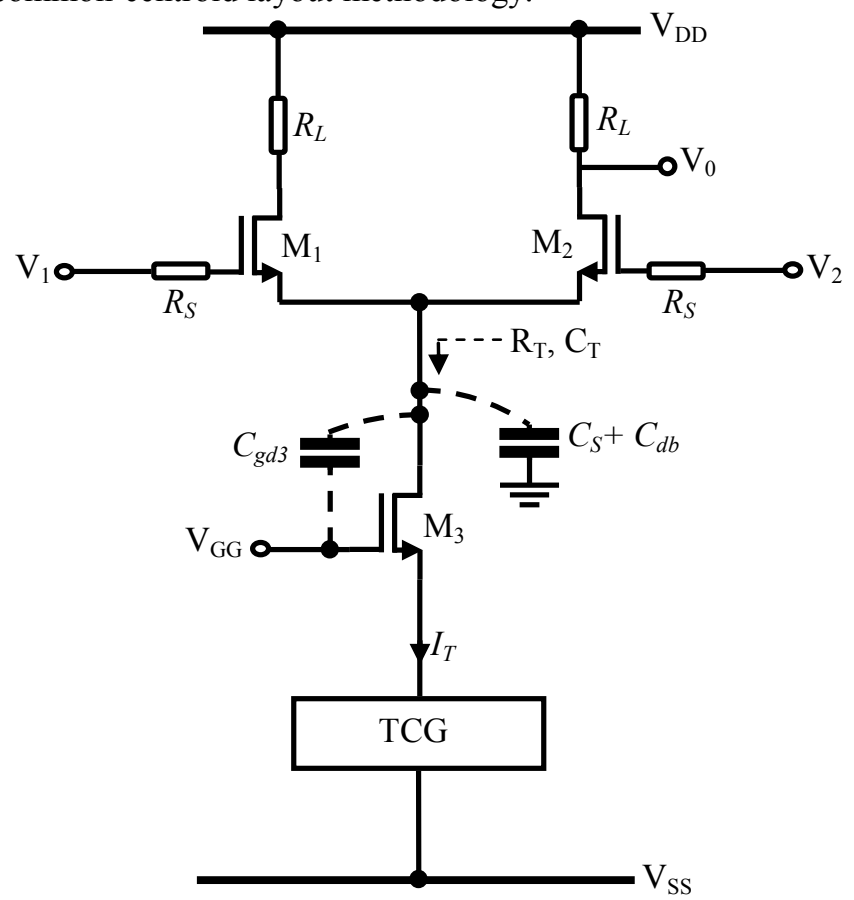

Fig. 1. Typical CMOS differential amplifier circuit $R_{T}, C_{T}, C_{d g 3}, C_{S}$ and $C_{d b}$ are defined in the text 
$\mathrm{M}_{3}$ is in the common-gate connection with its gate connected to $D C$ voltage $\mathrm{V}_{\mathrm{GG}}$, where $\mathrm{V}_{\mathrm{DD}}>\mathrm{V}_{\mathrm{GG}}>\mathrm{V}_{\mathrm{Ss}}$. The drain current $I_{T}$ is supplied by the Tail Current Generator (TCG) connected to its source. TCG can be merely a resistor but is often a current generator in order to increase the incremental output resistance, $R_{T}$, seen looking into the drain of $\mathrm{M}_{3}$. This needs to be a high value for a low common-mode gain and hence a high CMRR at low frequencies. TCG will have an unavoidable nodal capacitance, $C_{T}$, associated with it. This needs to be low to maximize the CMRR bandwidth.

The amplifier inputs $v_{1}$ and $v_{2}$ and, in this particular case, the single output $v_{0}$ can each be considered to comprise two components, (i) a differential-mode component, $v_{d}$, and (ii) a common-mode component, $v_{c}$.

Thus,

$$
\begin{aligned}
& v_{1}=v_{c}+\frac{1}{2} v_{d} \\
& v_{2}=v_{c}-\frac{1}{2} v_{d}
\end{aligned}
$$

and,

$$
v_{0}=A_{d} \mathrm{v}_{\mathrm{d}}+A_{c} v_{c}
$$

The differential-mode voltage gain, $A_{d}$, and the commonmode voltage gain, $A_{c}$, are defined as follows,

$$
A_{d}=\frac{v_{o}}{v_{d}} \mid \text { With } v_{c}=0
$$

and,

$$
A_{c}=\frac{v_{o}}{v_{c}} \mid \text { With } v_{d}=0
$$

The CMRR, denoted here by the symbol $\varrho$, is defined as,

$$
\text { CMRR }=\varrho=\left|\frac{A_{d}}{A_{c}}\right|
$$

$A_{d}$, and $A_{c}$ are frequency-dependent, hence so too is $\varrho$. For the determination of $A_{d}$ the differential-drive considered, the long-tailed pair, formed by $\mathrm{M}_{1}, \mathrm{M}_{2}$ and its common-source tail current $I_{T}$, is imagined to be reconfigured as two commonsource half circuits. By inspection, $A_{d}$ has a $D C$ and very-lowfrequency gain of magnitude $g_{m} \cdot R_{L}^{\prime} / 2$, where $g_{m}$ is the transconductance of each of $\mathrm{M}_{1}, \mathrm{M}_{2}$ at a $D C$ operating current $I_{T} / 2$ and $R_{L}^{\prime}=R_{L} / / r_{d s}$ ( $r_{d s}$ being the drain-source incremental resistance of each of the MOSFETs ). Based on the work of [5], with appropriately modified notation, $A_{d}$ exhibits a dominant pole at a radian frequency $\omega_{p c}$.

$$
\omega_{p d}=\frac{1}{R_{S} C_{g s}+R_{S} C_{g d}\left(1+g_{m} R_{L}^{\prime}\right)+R_{L}^{\prime}\left(C_{L}+C_{g d}\right)}
$$

In this equation, $C_{g s}$ and $C_{g d}$ have their usual MOSFET significance and $C_{L}$ is the load capacitance existing at the drain of each of $\mathrm{M}_{1}$, and of $\mathrm{M}_{2}$.

For the determination of $A_{C}$, the circuit is regarded as two separate stages, each having a source load $2 Z_{T}$ (where, $Z_{T}=R_{T} / / C_{T}$ and the drain load $Z_{L}=R_{L}^{\prime} / / C_{L}$ )

Hence, in terms of the complex frequency variable $s$,

$A_{c}(s)=-\frac{Z_{L}}{2 Z_{T}}=-\frac{R_{L}^{\prime}}{2 R_{T}}=\frac{\left(1+s R_{T} C_{T}\right)}{\left(1+s R_{L}^{\prime} C_{L}\right)}$

Thus, $A_{c}$ exhibits a $D C$ and very low-frequency gain of magnitude $-R_{L}^{\prime} / 2 R_{T}$, and a pole at a radian frequency $\omega_{p c}$ and

zero at $\omega_{z c}$,

$$
\begin{gathered}
\omega_{p c}=\frac{1}{R_{L}^{\prime} C_{L}} \\
\omega_{z c}=\frac{1}{R_{T} C_{T}}
\end{gathered}
$$

$C_{T}$ comprises three capacitances, $C_{g d 3}$ and $C_{d b 3}$, respectively the drain-gate and drain-substrate capacitance of $\mathrm{M}_{3}$, together with $C_{S}$ the stray capacitance at its drain, thus,

$C_{T}=C_{S}+C_{d b 3}+C_{g b 3}$

The zero at $\omega_{z c}$ on the plot of $A c$ versus $\omega$ now appears as a pole on the CMRR-frequency plot in addition to the pole at $\omega_{p c}$, and the pole of $\omega_{p c}$ on the $A c$ plot appears as a zero on the CMRR plot. The nature of existing circuit designs is such that $\omega_{p c} \gg \omega_{z c}$, because $R_{T} \gg R_{L}^{\prime}, \quad R_{S}$. Furthermore, $\omega_{p c} \gg \omega_{z c}$ because, even if $\left(C_{T}+C_{g d 2}\right)=10 C_{T}, R_{T} \gg R_{L}^{\prime}, R_{S}$ (e.g,. $\left.R_{T}>>100 R_{L}^{\prime}\right)$.

Consequently, $\omega_{z c}$ is the dominant pole on the CMRRfrequency plot, and effectively determines the CMRR $-3 \mathrm{~dB}$ bandwidth because of the relationship of $\omega_{z c}$ to $\omega_{p d}$ and $\omega_{p c}$. Thus, on a Bode plot of $\varrho$ versus $\omega, \varrho$ is constant at a value $\varrho_{o}=g_{m} \cdot R_{T}$ only up to a frequency $\omega_{z c}$. From the above discussion,

$\varrho_{o} \omega_{z c}=g_{m} / C_{T}$

So, for a specified and practically achievable $\varrho_{o}, \omega_{z c}$ is maximized by minimizing $C_{T}$. This can be done, as described below, by using a MOSFET variant of an elegant bipolar technique outlined by Baxandall and Swallow [6], but seemingly neglected in the literature until investigated in detail in recent years by Terzopoulos [7].

In passing, it is worth noting that the constancy of the product $\varrho_{o} \omega_{z c}$ with variation in $R_{T}$ is analogous to the constancy of the gain-bandwidth product in resistively loaded commonsource (and common-emitter) voltage amplifier stages. 
bandwidth, $\omega_{z c}$ as determined from the phase-shift corresponding to the $-3 \mathrm{~dB}$ points, is more than quadrupled.

In a third series of tests to see what happens when the design of Fig.1 was operated with same total current as the proposed design of Fig.2. $I_{T}$ in Fig.1 was $1.1 \mathrm{~mA}$, the current used for Fig.2. The resulting CMRR-frequency plot is curve $\mathrm{C}$, which shows a lower cut-off frequency than curves $\mathrm{A}$ and $\mathrm{B}$. Curves $\mathrm{A}, \mathrm{B}$ and $\mathrm{C}$ all have slightly different values of $\varrho_{o}$. This is because the different $D C$ bias currents in the tests leads to differing values of the small signal parameters $g_{m}$ and $r_{d s}$ of the MOSFETs (and hence differing values for $R_{T}$ ). Curves $\mathrm{A}$ and $\mathrm{C}$ are for the same $C_{T}$, but the effective $R_{T}$ of $C_{T}$ is greater than that of $\mathrm{A}$. In accordance with the discussion in section 2, above, this means a lower $\omega_{z c}$ but that the curves are coincident well above their cut-off frequencies.

It should be noted that the load resistors $\left(R_{L}\right)$ have been used to simplify the discussion and analysis. The resistive loads gave a low frequency value of $A_{d}$ in the region of $60 \mathrm{~dB}$. In practice it is most likely that P-MOS transistors configured as active load current sources would be used instead to provide a considerably higher $A_{d}$. However, the method of increasing bandwidth of CMRR which has been presented here is still maintained with active loads in the drains of $\mathrm{M}_{1}$ and $\mathrm{M}_{2}$.

In this paper a MOSFET differential amplifier has been considered because of the growing importance of CMOS in analogue circuit design. However, depending on the application, bipolar transistors maybe employed, instead of MOSFETs, with $\mathrm{M}_{1}, \mathrm{M}_{2}, \mathrm{M}_{3}, \mathrm{M}_{4}$ being replaced by bipolar transistors $\mathrm{Q}_{1}, \mathrm{Q}_{2}, \mathrm{Q}_{3}, \mathrm{Q}_{4}$ respectively. Then the presence of $\mathrm{Q}_{4}$ not only partially compensates of the collector-base capacitance of $\mathrm{Q}_{3}$ but, as shown in [7], it also increases the incremental output resistance seen looking into its collector.

\section{Conclusions}

The addition of only one transistor, plus associated bias circuitry, into a conventional source-coupled differential amplifier has been shown to extend the CMRR bandwidth substantially. This performance improvement is a result of local feedback reducing the source-coupled nodal capacitance of the differential pair. This technique is primarily applicable to an integrated circuit realization of the source-coupled differential amplifier because closely matched transistors operating at the same temperature are essential for satisfactory operation.

\section{References}

[1] S. Franco, : 'Design with Operational Amplifiers and Analog Integrated Circuits', McGraw-Hill Higher Education, New York, 2002, Chapter 2, pp. $73-76$.

[2] S. Takagi, and T. Yanagisawa, : 'Improvement of Differential NIC Amplifier CMRR Using Balanced-type Constant C', Electronics Letters., 1966, Vol 25, Issue 2, pp. 153-154.

[3] A. A. Ciubotaru, : 'Technique for improving high-frequency CMRR of emitter-coupled differential pairs', Electronics Letters., 2002, Vol 38, Issue 17, pp. 943-944.

[4] P. A. Dal Fabbro, and C. A. Dos Reis Filh, : 'An Integrated CMOS Instrumentation Amplifier with Improved CMRR', Integrated Circuits and Systems Design., 2002, Proceedings $15^{\text {th }}$, pp. 57-61.

[5] P. R. Gray, P. J. Hurst, S. H. Lewis, and R. G. Meyer, : 'Analysis and Design of Analog Integrated Circuits', John Wiley \& Sons, Inc., Fifth Edition, New York, 2010, Chapter 9, p. 640.

[6] P. Baxandall, and E. E. Swallow, : 'Constant current source with unusually high internal resistance and good temperature stability', Electronic Letters., 1966, Vol 2, Issue 9, pp. 351-355.

[7] N. Terzopoulos, : 'High output current drive circuits for medical applications', Ph.D. Thesis, Oxford Brookes University, Oxford, 2006, Chapter 5, pp. 5.10-5.45.

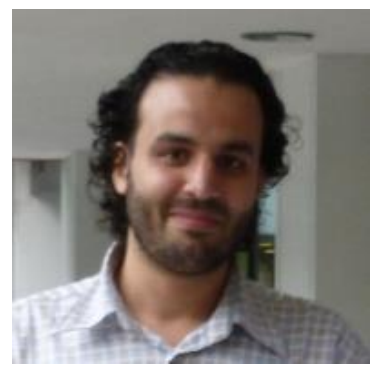

Mohamed Ben-Esmael received both his Bachelor's degree in Electronic Systems Design in 2009 and his Master's degree in Wireless Communication systems in 2010 from Oxford Brookes University.

$\mathrm{He}$ is currently working towards a $\mathrm{PhD}$ degree in the field of bioelectronic engineering within the Electronic Circuit and Systems Design and the Biomedical Imaging and Instrumentation Research Group at Oxford Brookes University, UK.

$\mathrm{He}$ is a member of the Institute of Electrical and Electronics Engineers (IEEE) and Institution of Engineering and Technology (IET). His research interests include bioelectronic circuits and systems and circuit techniques at high frequencies to achieve high output impedance.

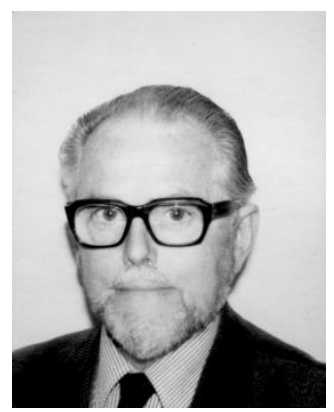

B. L. Hart received his B.Sc. in Physics from Queen Mary College, London University (UK) in 1951 and following military service, a Postgraduate Diploma in Electronics from Edinburgh, Scotland, in 1954. After seven years working on the design of radar displays in the research laboratories of the Marconi Company, he entered the teaching profession and subsequently became Head of Electronics Group in what is now the University of East London. On retirement, he became a part-time lecturer and consultant. 
In 2008 he completed his $\mathrm{PhD}$ thesis on 'Novel analogue electronic circuit designs exploiting device matching principles'.

Dr. Hart has authored over 100 publications and two undergraduate textbooks, 'Digital signal transmission: line circuit technology' (1987) and 'Introduction to Analogue Electronics' (1997).

His research interests include device modelling, precision analogue circuit design and pulse electronics. Dr. Hart, an IET member, is currently an honorary research fellow at Oxford Brookes University.

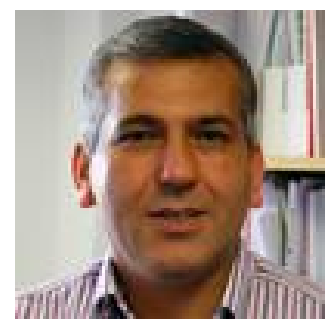

K. Hayatleh (SMIEEE, CEng, MIET) received his BEng in June 1992 from Oxford Brookes University and subsequent $\mathrm{PhD}$ in March 1996 from Oxford Brookes University in collaboration with Imperial College, London.

His research interests are in the area of electronic circuits and systems for RF and biomedical applications. Current projects are in transconductance amplifier design for high frequency RF applications, amplifier linearisation, current feedback op-amp development, RF LNA design, high accuracy voltage buffer and high output impedance current source design and a low-voltage high-speed CMOS line driver for SerDes applications

He has over 90 publications in the above areas of research. He has been a keynote speaker at conferences in this area. Professor Hayatleh is currently the lead for electronics in the Biomedical Imaging and Instrumentation Research Team at Oxford Brookes University, UK.

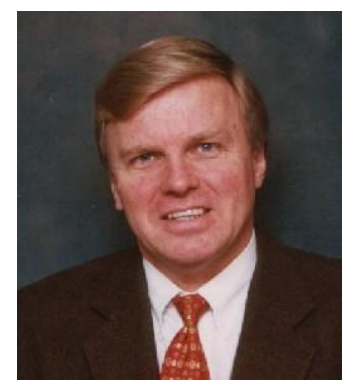

F. J. Lidgey completed his $\mathrm{PhD}$ at the University of Surrey in 1973 and after four years at the University of Newcastle, Australia returned to England in 1978 to join Oxford Brookes University, where he is now Emeritus Professor of Electronics.

His research interests within the Electronic Circuit and Systems Design Research Group and teaching are in the same area of analogue electronics. He has contributed to many novel circuit and system developments that fall under the general heading of currentmode analog design and published over 150 papers in this area.

In 1991 he was joint winner of the IEE's Rayleigh Best Book Prize for his contribution to, 'Analog Signal Processing: the Current-Mode Approach', published by Peter Peregrinus Limited. Also, he has a long term interest in using computers in education and in recent years has focused on the development of computer-based-learning for teaching undergraduate electronics. 